\title{
TOPOLOGICAL PRESSURE VIA SADDLE POINTS
}

\author{
KATRIN GELFERT AND CHRISTIAN WOLF
}

\begin{abstract}
Let $\Lambda$ be a compact locally maximal invariant set of a $C^{2}$ diffeomorphism $f: M \rightarrow M$ on a smooth Riemannian manifold $M$. In this paper we study the topological pressure $P_{\text {top }}(\varphi)$ (with respect to the dynamical system $f \mid \Lambda$ ) for a wide class of Hölder continuous potentials and analyze its relation to dynamical, as well as geometrical, properties of the system. We show that under a mild non-uniform hyperbolicity assumption the topological pressure of $\varphi$ is entirely determined by the values of $\varphi$ on the saddle points of $f$ in $\Lambda$. Moreover, it is enough to consider saddle points with "large" Lyapunov exponents. We also introduce a version of the pressure for certain non-continuous potentials and establish several variational inequalities for it. Finally, we deduce relations between expansion and escape rates and the dimension of $\Lambda$. Our results generalize several well-known results to certain non-uniformly hyperbolic systems.
\end{abstract}

\section{INTRODUCTION}

1.1. Motivation. In the geometric and ergodic-theoretical aspects of the theory of dynamical systems, the so-called thermodynamic formalism, which was originally developed by theoretical physicists, has become a powerful tool during the last three decades. The main object in this theory is the topological pressure, i.e. a particular functional on the space of observables, that encodes several important quantities of the underlying dynamical system. For example, pressure can be applied to obtain information about Lyapunov exponents, dimension, multifractal spectra, natural invariant measures, etc. (see for instance [12] and the references therein). In particular, in the case of hyperbolic systems Bowen and Ruelle established in their pioneer works deep connections between topological pressure and periodic points, Hausdorff dimension and the characterization of attractors. The main purpose of this paper is to generalize some of these results to the case of certain non-uniformly hyperbolic systems. One key idea in our approach is to apply a theory developed by Katok, and Katok and Mendoza concerning the existence of hyperbolic horseshoes in the presence of hyperbolic ergodic invariant probability measures. We show that under the assumption of mild non-uniform hyperbolicity the topological pressure of

Received by the editors September 8, 2006.

2000 Mathematics Subject Classification. Primary 37D25, 37D35; Secondary 37C25, 37C40, $37 \mathrm{C} 45$.

Key words and phrases. Topological pressure, $C^{2}$-diffeomorphism, saddle points, invariant measures, Hausdorff dimension.

The research of the first author was supported by the Deutsche Forschungsgemeinschaft. The research of the second author was supported in part by the National Science Foundation under Grant No. EPS-0236913 and matching support from the State of Kansas through Kansas Technology Enterprise Corporation. 
a Hölder continuous potential is entirely determined by the values of the potential on the saddle points. Moreover, it is sufficient to consider only saddle points with "large" absolute value of the Lyapunov exponents. Staying in this setting and by using saddle points, we propose a pressure for non-continuous potentials and derive several variational inequalities for it. Finally, we establish relations between the attraction properties of the system and the dimension of the invariant set.

1.2. Statement of the results. We now describe our results in more detail. Let $M$ be a smooth Riemannian manifold and let $\Lambda \subset M$ be a compact locally maximal invariant set of a $C^{2}$-diffeomorphism $f: M \rightarrow M$. Given $\varphi \in C(\Lambda, \mathbb{R})$ we denote by $P_{\text {top }}(\varphi)$ the topological pressure of the potential $\varphi$; see Section 2 for the definition and details. Let $C^{f}(\Lambda, \mathbb{R})$ be defined as in Section 3. Roughly speaking a potential $\varphi$ belongs to $C^{f}(\Lambda, \mathbb{R})$ if $P_{\text {top }}(\varphi)$ can be approximated by free energies of measures whose absolute values of the Lyapunov exponents are uniformly bounded away from zero, and $\varphi$ has no equilibrium state with zero entropy. We denote the corresponding uniform bound by $\delta(\varphi)$. Again we refer to Section 3 for the details. Given $n \in \mathbb{N}$ let $\operatorname{Fix}\left(f^{n}\right)$ denote the set of fixed points of $f^{n}$, and let $\operatorname{SFix}\left(f^{n}\right) \subset \operatorname{Fix}\left(f^{n}\right)$ denote the saddle points in $\operatorname{Fix}\left(f^{n}\right)$. Moreover, for $0<\alpha, 0<c \leq 1$, let $\operatorname{SFix}\left(f^{n}, \alpha, c\right)$ be defined as in (9). Roughly speaking, $\operatorname{SFix}\left(f^{n}, \alpha, c\right)$ are those saddle points for which the infimum norm of the derivative restricted to the stable/unstable spaces grows uniformly at an exponential rate at least $\alpha$. Therefore, if $x \in \operatorname{SFix}\left(f^{n}, \alpha, c\right)$, then all Lyapunov exponents of $x$ have absolute value greater than or equal to $\alpha$. Let $\operatorname{SPer}(f)$ denote the set of all saddle points of $f$ in $\Lambda$. The sets $\operatorname{SFix}\left(f^{n}, \alpha, c\right)$ provide a natural filtration of $\operatorname{SPer}(f)$; in particular,

$$
\operatorname{SPer}(f)=\bigcup_{\alpha>0} \bigcup_{c>0} \bigcup_{n=1}^{\infty} \operatorname{SFix}\left(f^{n}, \alpha, c\right) .
$$

For $\varphi \in C(\Lambda, \mathbb{R})$ we define

$$
\mathrm{P}_{\mathrm{SP}}(\varphi, \alpha, c)=\limsup _{n \rightarrow \infty} \frac{1}{n} \log \left(\sum_{x \in \operatorname{SFix}\left(f^{n}, \alpha, c\right)} \exp S_{n} \varphi(x)\right),
$$

where $S_{n} \varphi(x)=\sum_{k=0}^{n-1} \varphi\left(f^{k}(x)\right)$. Our main result shows that in the case of Hölder continuous potentials in $C^{f}(\Lambda, \mathbb{R})$ the topological pressure is entirely determined by the values of $\varphi$ on the saddle points. More precisely, we have the following result.

Theorem 1. Let $f: M \rightarrow M$ be a $C^{2}$-diffeomorphism and let $\Lambda \subset M$ be a compact locally maximal $f$-invariant set. Let $\varphi \in C^{f}(\Lambda, \mathbb{R})$ be a Hölder continuous potential and let $0<\alpha<\delta(\varphi)$. Then

$$
P_{\text {top }}(\varphi)=\lim _{c \rightarrow 0} P_{\mathrm{SP}}(\varphi, \alpha, c) .
$$

It follows from Ruelle's inequality that in the case of surface diffeomorphisms Theorem 1 holds for a more general class of potentials, namely (2) is true for all Hölder continuous potentials admitting no equilibrium state with zero measuretheoretic entropy. We note that the hypothesis $\varphi \in C^{f}(\Lambda, \mathbb{R})$ can, in general, not be omitted in Theorem 1. A simple counterexample is given in Section 3 (see Example 2). Theorem 1 is even new for uniformly hyperbolic sets. Indeed, Bowen proved a version of (2) in the case of hyperbolic sets by considering all saddle points. On the other hand, our result shows that it is already sufficient to consider saddle 
points with "large" Lyapunov exponents. We emphasize, however, that the main application of Theorem 1 is that to non-uniformly hyperbolic systems. Theorem 1 is a generalization of a result of Chung and Hirayama [8]. They considered the entropy of diffeomorphisms on compact surfaces, i.e. $\operatorname{dim} M=2, \Lambda=M$ and $\varphi=0$, and we use some of their ideas in our approach. It should be noted that on higher dimensional manifolds the potential $\varphi=0$ does not always belong to $C^{f}(\Lambda, \mathbb{R})$; see Example 1 in Section 3 .

We also consider a version of pressure for non-continuous potentials. As a particular case we study the volume expansion of the derivative restricted to the expanding subbundle $E^{u}$ defined by

$$
\varphi^{u}(x)=-\log |\operatorname{det} D f(x)| E_{x}^{u} \mid .
$$

In general, the subbundle $E^{u}$ can only be defined over a particular subset of $\Lambda$, and $\varphi^{u}$ does not extend to a continuous function on $\Lambda$. Nevertheless, since $E_{x}^{u}$ exists for all saddle points $x$, we still can define

$$
P_{\mathrm{SP}}\left(\varphi^{u}\right)=\lim _{\alpha \rightarrow 0} \lim _{c \rightarrow 0} \mathrm{P}_{\mathrm{SP}}\left(\varphi^{u}, \alpha, c\right) .
$$

We call $P_{\mathrm{SP}}\left(\varphi^{u}\right)$ the volume pressure of $f$. Note that if $\Lambda$ is a locally maximal uniformly hyperbolic set such that $f \mid \Lambda$ is topologically mixing, then by a classical result of Bowen $P_{\mathrm{SP}}\left(\varphi^{u}\right)$ coincides with $P_{\text {top }}\left(\varphi^{u}\right)$. We show in Corollary 2 that in the case of general sets $\Lambda$ which contain at least one saddle point we have

$$
P_{\mathrm{SP}}\left(\varphi^{u}\right) \leq \sup _{\mu}\left(h_{\mu}(f)+\int_{\Lambda} \varphi^{u} d \mu\right)
$$

where the supremum is taken over all $f$-invariant probability measures $\mu$ of saddle type. We note that even in the case of uniformly hyperbolic sets (assuming that $f \mid \Lambda$ is not topologically mixing) inequality (3) can be strict.

The volume pressure can be applied to characterize certain attraction properties on $\Lambda$. Young showed in [15] that

$$
\sup _{\mu}\left(h_{\mu}(f)+\int_{\Lambda} \varphi^{u} d \mu\right) \leq \underline{E}(V) \leq \bar{E}(V) \leq 0
$$

where the supremum is taken over all ergodic invariant measures $\mu$, and $\underline{E}(V)$ $(\bar{E}(V))$ denotes the lower (upper) escape rate from a neighborhood $V$ of $\Lambda$ (see (33) for the precise definition).

In the case of a locally maximal topologically mixing hyperbolic set $\Lambda$ Bowen [5] showed (also using joint results with Ruelle [6]) that $P_{\mathrm{SP}}\left(\varphi^{u}\right)=\bar{E}(V)$; in particular, all the inequalities in (3) and (4) are identities and the corresponding quantity coincides with $P_{\text {top }}\left(\varphi^{u}\right)$. Moreover, he proved that $\Lambda$ is an attractor if and only if $P_{\text {top }}\left(\varphi^{u}\right)=0$. The latter result has recently been extended in [13] by adding the a priori weaker but still equivalent condition $\operatorname{dim}_{\mathrm{H}} W^{s}(\Lambda)=\operatorname{dim} M$. Here $\operatorname{dim}_{H} W^{s}(\Lambda)$ denotes the Hausdorff dimension of the stable set of $\Lambda$. Using ideas of [13] we show for a general set $\Lambda$ that if $\bar{E}(V)<0$, then the upper box dimension $\overline{\operatorname{dim}}_{\mathrm{B}} \Lambda$ of $\Lambda$ is strictly smaller than $\operatorname{dim} M$. More precisely, we derive in Theorem 4 an upper bound for $\overline{\operatorname{dim}}_{\mathrm{B}} \Lambda$ in terms of $\bar{E}(V)$ and the maximal asymptotic exponential expansion rate of $f$ on $\Lambda$. This bound is strictly smaller than $\operatorname{dim} M$ provided that $\bar{E}(V)<0$.

We now briefly describe the content of the paper. In Section 2 we review several concepts and results from smooth ergodic theory and introduce various notions of 
pressure. Section 3 is devoted to the statements in our main result of Theorem 1 . In Section 4 we introduce a version of the pressure for non-continuous potentials. We study this pressure in the particular case of the potential $\varphi^{u}=-\log |\operatorname{det} D f| E^{u} \mid$ and derive several variational inequalities for it. Finally, in Section 5 we discuss relations between the escape rates and the dimension of $\Lambda$.

\section{Preliminaries}

2.1. Notions from smooth ergodic theory. Let $M$ be a smooth Riemannian manifold and let $f: M \rightarrow M$ be a $C^{2}$-diffeomorphism. We consider a compact locally maximal $f$-invariant set $\Lambda \subset M$. Here locally maximal means that there exists an open neighborhood $U \subset M$ of $\Lambda$ such that $\Lambda=\bigcap_{n \in \mathbb{Z}} f^{n}(U)$. Note that in particular, if $M$ is compact, the case $\Lambda=M$ fits within this setup. To avoid trivialities we will always assume that $h_{\text {top }}(f \mid \Lambda)>0$, where $h_{\text {top }}$ denotes the topological entropy of the map. This rules out the case that $\Lambda$ is only a periodic orbit. Given $x \in \Lambda$ and $v \in T_{x} M \backslash\{0\}$, we define the forward Lyapunov exponent of $v$ at $x$ (with respect to $f$ ) by

$$
\lambda^{+}(x, v) \stackrel{\text { def }}{=} \limsup _{n \rightarrow \infty} \frac{1}{n} \log \left\|D f^{n}(x)(v)\right\| .
$$

Analogously, we define a Lyapunov exponent $\lambda^{-}(x, v)$ for negative time by replacing the map $f$ in (5) with $f^{-1}$, which is called the backward Lyapunov exponent of $v$ at $x$ (with respect to $f$ ). Let us now assume that $x \in \Lambda$ is a Lyapunov regular point of $f$ (see [3] for the definition and details on Lyapunov regularity). Then there exist a positive integer $s(x) \leq \operatorname{dim} M$ and a $D f$-invariant splitting

$$
T_{x} M=\bigoplus_{i=1}^{s(x)} E_{x}^{i}
$$

such that for all $i=1, \ldots, s(x)$ and $v \in E_{x}^{i} \backslash\{0\}$ we have

$$
\lim _{n \rightarrow \pm \infty} \frac{1}{|n|} \log \left\|D f^{n}(x)(v)\right\|=\lambda_{i}^{+}(x)=-\lambda_{i}^{-}(x) \stackrel{\text { def }}{=} \lambda_{i}(x),
$$

with uniform convergence on $\left\{v \in E_{x}^{i}:\|v\|=1\right\}$. We will count the values of the Lyapunov exponents $\lambda_{i}(x)$ with their multiplicities, i.e. we consider the numbers

$$
\lambda_{1}(x) \leq \cdots \leq \lambda_{\operatorname{dim} M}(x) .
$$

Let $\mathcal{M}$ denote the set of all Borel $f$-invariant probability measures on $\Lambda$ endowed with weak* topology. This makes $\mathcal{M}$ a compact convex space. Moreover, let $\mathcal{M}_{\mathrm{E}} \subset$ $\mathcal{M}$ be the subset of ergodic measures. By Oseledec's theorem, given $\mu \in \mathcal{M}$ the set of Lyapunov regular points has full measure and $\lambda_{i}(\cdot)$ is $\mu$-measurable. We denote by

$$
\lambda_{i}(\mu) \stackrel{\text { def }}{=} \int \lambda_{i}(x) d \mu(x)
$$

the Lyapunov exponents of the measure $\mu$. Note that if $\mu \in \mathcal{M}_{\mathrm{E}}$, then $\lambda_{i}($.$) is$ constant $\mu$-a.e., and therefore the corresponding value coincides with $\lambda_{i}(\mu)$. We say that $\mu \in \mathcal{M}$ is a hyperbolic measure if $\mu$ has non-zero Lyapunov exponents. Set

$$
\chi(\mu) \stackrel{\text { def }}{=} \min _{i=1, \ldots, \operatorname{dim} M}\left|\lambda_{i}(\mu)\right| .
$$


In particular, if there is $1 \leq l=l(\mu)<\operatorname{dim} M$ such that

$$
\lambda_{l}(\mu)<0<\lambda_{l+1}(\mu),
$$

we say that $\mu$ is of saddle type. It follows from Ruelle's inequality that for a surface diffeomorphism every hyperbolic measure $\mu$ with positive measure-theoretic entropy $h_{\mu}(f)$ is of saddle type. We denote by $\operatorname{Fix}(f)$ the set of fixed points of $f$. Moreover, we denote by $\operatorname{Per}(f)=\bigcup_{n} \operatorname{Fix}\left(f^{n}\right)$ the set of periodic points of $f$. For $x \in \operatorname{Fix}\left(f^{n}\right)$ we have that $\lambda_{i}(x)=\frac{1}{n} \log \left|\delta_{i}\right|$, where $\delta_{i}$ are the eigenvalues of $D f^{n}(x)$. We call a periodic point $x$ a saddle point if there is $1 \leq l=l(x)<\operatorname{dim} M$ with $\lambda_{l}(x)<0<\lambda_{l+1}(x)$. Let $\operatorname{SFix}\left(f^{n}\right)$ denote the fixed points of $f^{n}$ which are saddle points. Hence, $\operatorname{SPer}(f)=\bigcup_{n} \operatorname{SFix}\left(f^{n}\right)$ is the set of all saddle points.

We say that a compact $f$-invariant set $K \subset M$ is a hyperbolic set if there exists a continuous $D f$-invariant splitting of the tangent bundle $T_{K} M=E^{s} \oplus E^{u}$ and constants $c>0$ and $\lambda \in(0,1)$ such that

$$
\begin{gathered}
\left\|D f^{k}(x)(v)\right\| \leq c \lambda^{k}\|v\| \text { for all } v \in E_{x}^{s}, \\
\left\|D f^{-k}(x)(v)\right\| \leq c \lambda^{k}\|v\| \text { for all } v \in E_{x}^{u}
\end{gathered}
$$

for all $x \in K$ and all $k \in \mathbb{N}$. For convenience we sometimes also refer to relative compact sets satisfying (7) as hyperbolic sets.

For $x \in \operatorname{SFix}\left(f^{n}\right)$ we define $E_{x}^{s}\left(E_{x}^{u}\right)$ to be the direct sum of the eigenspaces corresponding to eigenvalues of $D f^{n}(x)$ with norm smaller than 1 (larger than 1). It is easy to see that there exists $0<c \leq 1, c=c(x)$, such that for all integers $k \geq 0$ and $0 \leq i \leq n-1$

$$
\begin{aligned}
c e^{k \lambda_{l+1}(x)} & \leq\left\|D f^{k}\left(f^{i}(x)\right)(v)\right\|, \\
c e^{-k \lambda_{l}(x)} & \leq\left\|D f^{-k}\left(f^{i}(x)\right)(w)\right\|
\end{aligned}
$$

whenever $v \in E_{f^{i}(x)}^{u}$ with $\|v\|=1$ and $w \in E_{f^{i}(x)}^{s}$ with $\|w\|=1$. For $0<\alpha$, $0<c \leq 1$, and $n \in \mathbb{N}$ we set

(9) $\operatorname{SFix}\left(f^{n}, \alpha, c\right) \stackrel{\text { def }}{=}\left\{x \in \operatorname{SFix}\left(f^{n}\right):\left\|\left(D f^{-k}\left(f^{i}(x)\right) \mid E^{s}\right)^{-1}\right\|^{-1} \geq c e^{k \alpha}\right.$,

$$
\left.\left\|\left(D f^{k}\left(f^{i}(x)\right) \mid E^{u}\right)^{-1}\right\|^{-1} \geq c e^{k \alpha} \text { for all } k \geq 1 \text { and } 0 \leq i \leq n-1\right\} .
$$

Thus, if $\alpha \geq \alpha^{\prime}, c \geq c^{\prime}$, then

$$
\operatorname{SFix}\left(f^{n}, \alpha, c\right) \subset \operatorname{SFix}\left(f^{n}, \alpha^{\prime}, c^{\prime}\right)
$$

and

$$
\operatorname{SPer}(f)=\bigcup_{\alpha>0} \bigcup_{c>0} \bigcup_{n=1}^{\infty} \operatorname{SFix}\left(f^{n}, \alpha, c\right) .
$$

Lemma 1. Let $\alpha, c>0$ be fixed. Then $x \mapsto E_{x}^{s / u}$ are continuous maps on $L=$ $\bigcup_{n=1}^{\infty} \operatorname{SFix}\left(f^{n}, \alpha, c\right)$ which extend continuously to the closure of $L$.

Proof. It suffices to notice that for $y \in L$ we have

$$
\left\|D f^{k}(y)\left|E^{s}\|\| D f^{-k}\left(f^{k}(y)\right)\right| E^{u}\right\| \leq \frac{1}{c^{2}} e^{-2 k \alpha} \leq \frac{1}{2}
$$

whenever $k \geq \frac{1}{2 \alpha} \log \frac{2}{c^{2}}$. This means that the splitting of the tangent space $T_{L} M=$ $E^{s} \oplus E^{u}$ is $k$-dominated for any such $k$ (see [4] for details on dominated splittings). By $f$-invariance of $L$ the statement follows from [4, Lemma 1.4]. 
We now present some results concerning non-uniformly hyperbolic systems developed by Katok and Mendoza; see [9] in the case of surface diffeomorphisms and [3] in the general case. Let $\mu \in \mathcal{M}_{E}$ be a hyperbolic measure with positive measure-theoretic entropy. Then

$$
h_{\mu}(f) \leq \limsup _{n \rightarrow \infty} \frac{1}{n} \log \operatorname{cardFix}\left(f^{n}\right) .
$$

Moreover, for all $\varepsilon>0$ we have

$$
0<h_{\mu}(f) \leq \limsup _{n \rightarrow \infty} \frac{1}{n} \log \operatorname{card}\left\{x \in \operatorname{SFix}\left(f^{n}\right): \chi(x) \geq \chi(\mu)-\varepsilon\right\},
$$

where $\chi(x)=\min _{j=1, \ldots, \operatorname{dim} M}\left|\lambda_{j}(x)\right|$. Furthermore, there exist positive constants $\alpha_{0}$ and $c_{0}$ such that $\operatorname{SFix}\left(f^{n}, \alpha, c\right) \neq \emptyset$ for all $\alpha \leq \alpha_{0}, c \leq c_{0}$, and infinitely many $n \in \mathbb{N}$.

2.2. Various pressures. Next, we introduce a version of topological pressure which is entirely determined by the values of the potential on the saddle points.

Let us first recall the classical topological pressure. Let $(\Lambda, d)$ be a compact metric space and let $f: \Lambda \rightarrow \Lambda$ be a continuous map. For $n \in \mathbb{N}$ we define a new metric $d_{n}$ on $\Lambda$ by $d_{n}(x, y)=\max _{k=0, \ldots, n-1} d\left(f^{k}(x), f^{k}(y)\right)$. A set of points $\left\{x_{i}: i \in I\right\} \subset \Lambda$ is called $(n, \varepsilon)$-separated (with respect to $f$ ) if $d_{n}\left(x_{i}, x_{j}\right)>\varepsilon$ holds for all $x_{i}, x_{j}$ with $x_{i} \neq x_{j}$. Fix for all $\varepsilon>0$ and all $n \in \mathbb{N}$ a maximal (with respect to the inclusion) $(n, \varepsilon)$-separated set $F_{n}(\varepsilon)$. The topological pressure (with respect to $f \mid \Lambda)$ is a mapping $P_{\text {top }}(f \mid \Lambda,):. C(\Lambda, \mathbb{R}) \rightarrow \mathbb{R}$ defined by

$$
P_{\text {top }}(f \mid \Lambda, \varphi) \stackrel{\text { def }}{=} \lim _{\varepsilon \rightarrow 0} \limsup _{n \rightarrow \infty} \frac{1}{n} \log \left(\sum_{x \in F_{n}(\varepsilon)} \exp S_{n} \varphi(x)\right),
$$

where

$$
S_{n} \varphi(x) \stackrel{\text { def }}{=} \sum_{k=0}^{n-1} \varphi\left(f^{k}(x)\right) .
$$

The topological entropy of $f$ on $\Lambda$ is defined by $h_{\text {top }}(f \mid \Lambda)=P_{\text {top }}(f \mid \Lambda, 0)$. We simply write $P_{\text {top }}(\varphi)$ and $h_{\text {top }}(f)$ if there is no confusion about $f$ and $\Lambda$. Note that the definition of $P_{\text {top }}(\varphi)$ does not depend on the choice of the sets $F_{n}(\varepsilon)$ (see [14]). The topological pressure satisfies the following variational principle:

$$
P_{\text {top }}(\varphi)=\sup _{\nu \in \mathcal{M}}\left(h_{\nu}(f)+\int_{\Lambda} \varphi d \nu\right) .
$$

Furthermore, the supremum in (14) can be replaced by the supremum taken only over all $\nu \in \mathcal{M}_{\mathrm{E}}$. We now introduce a pressure which is entirely defined by the values of $\varphi$ on the saddle points. Let $\varphi \in C(\Lambda, \mathbb{R})$ and let $0<\alpha, 0<c \leq 1$. Define

$$
Q_{\mathrm{SP}}(\varphi, \alpha, c, n) \stackrel{\text { def }}{=} \sum_{x \in \operatorname{SFix}\left(f^{n}, \alpha, c\right)} \exp S_{n} \varphi(x)
$$

if $\operatorname{SFix}\left(f^{n}, \alpha, c\right) \neq \emptyset$ and

$$
Q_{\mathrm{SP}}(\varphi, \alpha, c, n) \stackrel{\text { def }}{=} \exp \left(n \min _{x \in \Lambda} \varphi(x)\right)
$$


otherwise. Furthermore, we define

$$
P_{\mathrm{SP}}(\varphi, \alpha, c) \stackrel{\text { def }}{=} \limsup _{n \rightarrow \infty} \frac{1}{n} \log Q_{\mathrm{SP}}(\varphi, \alpha, c, n) .
$$

It follows from the definition that if $\operatorname{SFix}\left(f^{n}, \alpha, c\right) \neq \emptyset$ for some $n \in \mathbb{N}$, then this is true already for infinitely many $n \in \mathbb{N}$. Therefore, in the case when $\operatorname{SFix}\left(f^{n}, \alpha, c\right) \neq$ $\emptyset$ for some $n \in \mathbb{N}$, then $P_{\mathrm{SP}}(\varphi, \alpha, c)$ is entirely determined by the values of $\varphi$ on $\bigcup_{n \in \mathbb{N}} \operatorname{SFix}\left(f^{n}, \alpha, c\right)$.

The following classical result shows that in the case of hyperbolic sets the topological pressure of a Hölder continuous potential is entirely determined by its values on the saddle orbits. We only sketch its proof and refer to [10] for full details.

Proposition 1. Let $f: M \rightarrow M$ be a $C^{2}$-diffeomorphism and let $K \subset M$ be a compact hyperbolic set of $f$. Let $\varphi \in C(K, \mathbb{R})$ be a Hölder continuous potential. Then

$$
\limsup _{n \rightarrow \infty} \frac{1}{n} \log \left(\sum_{x \in \operatorname{Fix}\left(f^{n}\right) \cap K} \exp S_{n} \varphi(x)\right) \leq P_{\text {top }}(f \mid K, \varphi) .
$$

Furthermore, if $f \mid K$ satisfies the specification property, then we have equality in (15), and the limit superior is in fact a limit.

Proof. Since $K$ is a hyperbolic set the map $f \mid K$ is expansive. If $\delta$ is the expansivity constant, then for every $n \in \mathbb{N}$ and every $0<\varepsilon \leq \delta$ the set $\operatorname{Fix}\left(f^{n}\right)$ is $(n, \varepsilon)$ separated. Thus, the inequality (15) follows from the fact that the definition (12) can be replaced by the supremum taken over all $(n, \varepsilon)$-separated sets (see [14]). The equality in (15) is a direct consequence of [10, Proposition 20.3.3].

Remark. It follows from Proposition 1 and the Specification Theorem (see [10, Theorem 18.3.9]) that if $K$ is a locally maximal hyperbolic set such that $f \mid K$ is topologically mixing, then (15) is an identity.

\section{SADDLE POINTS AND TOPOLOGICAL PRESSURE}

In this section we study possible extensions of Proposition 1. In particular, we consider general locally maximal invariant sets $\Lambda$ without requiring hyperbolicity. Let $M$ be a smooth Riemannian manifold and let $f: M \rightarrow M$ be a $C^{2}$ diffeomorphism. Let $\Lambda \subset M$ be a compact locally maximal $f$-invariant set. Let $\varphi \in C(\Lambda, \mathbb{R})$. First, we investigate as to how periodic points with small absolute value of the Lyapunov exponents contribute to the pressure $P_{\text {top }}(\varphi)$. For $0<\alpha<\beta$ and $0<c \leq 1$ we define

$$
\begin{aligned}
& \operatorname{SFix}\left(f^{n},[\alpha, \beta], c\right)=\left\{x \in \operatorname{SFix}\left(f^{n}\right):\right. \\
& \quad c e^{k \alpha} \leq\left\|D f^{k}\left(f^{i}(x)\right)(v)\right\|,\left\|D f^{-k}\left(f^{i}(x)\right)(w)\right\| \leq c^{-1} e^{k \beta} \\
& \left.\quad \text { for all } k \geq 0 \text { and } 0 \leq i \leq n-1 \text { and all } v \in E_{x}^{u}, w \in E_{x}^{s}\right\} .
\end{aligned}
$$

It follows immediately from (8) that for every $x \in \operatorname{SFix}\left(f^{n},[\alpha, \beta], c\right)$ we have $\alpha \leq$ $\left|\lambda_{i}(x)\right| \leq \beta$ for all $i=1, \ldots, \operatorname{dim} M$. Furthermore,

$$
\operatorname{SFix}\left(f^{n}, \alpha, c\right)=\bigcup_{\beta>0} \operatorname{SFix}\left(f^{n},[\alpha, \beta], c\right)=\operatorname{SFix}\left(f^{n},\left[\alpha, \beta_{0}\right], c\right),
$$


where

$$
\beta_{0} \stackrel{\text { def }}{=} \max \left\{\log \|D f(x)\|, \log \left\|D f^{-1}(x)\right\|: x \in \Lambda\right\} .
$$

Define

$$
Q_{\mathrm{SP}}(\varphi,[\alpha, \beta], c, n)=\sum_{x \in \operatorname{SFix}\left(f^{n},[\alpha, \beta], c\right)} \exp S_{n} \varphi(x)
$$

if $\operatorname{SFix}\left(f^{n},[\alpha, \beta], c\right) \neq \emptyset$ and

$$
Q_{\mathrm{SP}}(\varphi,[\alpha, \beta], c, n)=\exp \left(n \min _{x \in \Lambda} \varphi(x)\right)
$$

otherwise. Furthermore, we define

$$
P_{\mathrm{SP}}(\varphi,[\alpha, \beta], c)=\limsup _{n \rightarrow \infty} \frac{1}{n} \log Q_{\mathrm{SP}}(\varphi,[\alpha, \beta], c, n) .
$$

It follows from the definition that if $\operatorname{SFix}\left(f^{n},[\alpha, \beta], c\right) \neq \emptyset$ for some $n \in \mathbb{N}$, then this is already true for infinitely many $n \in \mathbb{N}$. Therefore, in the case when $\operatorname{SFix}\left(f^{n},[\alpha, \beta], c\right) \neq \emptyset$ for some $n \in \mathbb{N}$ then $P_{\mathrm{SP}}(\varphi,[\alpha, \beta], c)$ is entirely determined by the values of $\varphi$ on $\bigcup_{n \in \mathbb{N}} \operatorname{SFix}\left(f^{n},[\alpha, \beta], c\right)$. We have the following:

Theorem 2. Let $f: M \rightarrow M$ be a $C^{2}$-diffeomorphism and let $\Lambda \subset M$ be a compact locally maximal $f$-invariant set. Let $0<\alpha<\beta$ and $0<c \leq 1$ such that $\operatorname{SFix}\left(f^{n},[\alpha, \beta], c\right) \neq \emptyset$ for some $n \in \mathbb{N}$. Let $\varphi \in C(\Lambda, \mathbb{R})$ be a Hölder continuous potential. Then

$$
P_{\mathrm{SP}}(\varphi,[\alpha, \beta], c) \leq \sup _{\nu}\left\{h_{\nu}(f)+\int_{\Lambda} \varphi d \nu\right\} \leq P_{\text {top }}(\varphi)
$$

where the supremum is taken over all $\nu \in \mathcal{M}_{\mathrm{E}}$ with $\alpha \leq\left|\lambda_{i}(\nu)\right| \leq \beta$ for all $i=$ $1, \ldots, \operatorname{dim} M$.

Proof. Let $0<\alpha<\beta$ and $0<c \leq 1$ such that $\operatorname{SFix}\left(f^{n},[\alpha, \beta], c\right) \neq \emptyset$ for some $n \in \mathbb{N}$. In particular, the supremum in (19) is not taken over the empty set. The right hand side inequality in (19) is a consequence of the variational principle. In order to prove the left hand side inequality set

$$
K=K_{\alpha, \beta, c} \stackrel{\text { def }}{=} \bigcup_{n=1}^{\infty} \operatorname{SFix}\left(f^{n},[\alpha, \beta], c\right) .
$$

The subspaces of the $D f$-invariant splitting $T_{x} M=E_{x}^{s} \oplus E_{x}^{u}$ vary continuously on the set $\bigcup_{n=1}^{\infty} \operatorname{SFix}\left(f^{n},[\alpha, \beta], c\right)$ and by Lemma 1 they can be extended continuously to $K$. It follows that $K$ is a hyperbolic set for $f$. Furthermore, for every $n \geq 1$ with $\operatorname{SFix}\left(f^{n},[\alpha, \beta], c\right) \neq \emptyset$ we have,

$$
\operatorname{Fix}\left(f^{n}\right) \cap K=\operatorname{SFix}\left(f^{n},[\alpha, \beta], c\right) .
$$

Therefore, Proposition 1 implies

$$
P_{\mathrm{SP}}(\varphi,[\alpha, \beta], c) \leq P_{\text {top }}(f \mid K, \varphi) .
$$

It follows from the variational principle that for every $\varepsilon>0$ there is a $\mu \in \mathcal{M}_{E}$ which is supported in $K$ such that

$$
P_{\text {top }}(f \mid K, \varphi)-\varepsilon \leq h_{\mu}(f)+\int_{K} \varphi d \mu \leq P_{\text {top }}(f \mid K, \varphi) .
$$


Since $\mu$ is ergodic we have that $\lambda_{i}(x)=\lambda_{i}(\mu)$ for $\mu$-almost every $x \in K$. It now follows from the continuity of $x \mapsto D f(x)$, the continuity of the extended splitting $T_{K} M=E^{s} \oplus E^{u}$ and the definition of $\operatorname{SFix}\left(f^{n},[\alpha, \beta], c\right)$ that $\alpha \leq\left|\lambda_{i}(x)\right| \leq \beta$ for all $x \in K$ and all $i=1, \ldots, \operatorname{dim} M$. We conclude that $\alpha \leq\left|\lambda_{i}(\mu)\right| \leq \beta$ for all $i=1, \ldots, \operatorname{dim} M$. Therefore, the left hand side inequality in (19) follows from (21) and (22). This completes the proof.

Proposition 2. Let $f: M \rightarrow M$ be a $C^{2}$-diffeomorphism and let $\Lambda \subset M$ be a compact locally maximal $f$-invariant set. Let $\varphi \in C(\Lambda, \mathbb{R})$ be a Hölder continuous potential. Then for all $\mu \in \mathcal{M}_{\mathrm{E}}$ with $h_{\mu}(f)>0$ and $\chi(\mu)>0$ and for all $0<\alpha<$ $\chi(\mu)$ we have

$$
h_{\mu}(f)+\int_{\Lambda} \varphi d \mu \leq \lim _{c \rightarrow 0} P_{\mathrm{SP}}(\varphi, \alpha, c) .
$$

Proof. Consider $\mu \in \mathcal{M}_{\mathrm{E}}$ with $h_{\mu}(f)>0$ and $\chi(\mu)>0$, and let $0<\alpha<\chi(\mu)$. It follows from the work of Katok and Mendoza (see e.g. [10, Chapter S.5] for the case of surface diffeomorphisms and [3] for the general case) that there exists a sequence $\left(\mu_{n}\right)_{n}$ of measures $\mu_{n} \in \mathcal{M}_{\mathrm{E}}$ supported on hyperbolic horseshoes $K_{n} \subset M$ (see [10] for the definition) such that $\mu_{n} \rightarrow \mu$ in the weak* topology, $h_{\mu_{n}}(f) \rightarrow h_{\mu}(f)$, and $\chi\left(\mu_{n}\right) \rightarrow \chi(\mu)$. In particular, for each $n \in \mathbb{N}$ there exist $m, s \in \mathbb{N}$ such that $f^{m} \mid K_{n}$ is conjugate to the full shift in $s$ symbols. Since $\Lambda$ is a compact locally maximal $f$-invariant set we can conclude that $K_{n} \subset \Lambda$ for all $n \in \mathbb{N}$. It follows that for every $0<\varepsilon<\chi(\mu)-\alpha$ there is a number $n=n(\varepsilon) \geq 1$ such that

$$
h_{\mu}(f)-\varepsilon<h_{\mu_{n}}(f) \text { and } \int_{\Lambda} \varphi d \mu-\varepsilon<\int_{\Lambda} \varphi d \mu_{n} .
$$

Moreover, there exists a number $c_{0}=c_{0}(n)$ with $0<c_{0}(n) \leq 1$ such that for every periodic point $x \in K_{n}$ and every $k \in \mathbb{N}$ we have

$$
\begin{gathered}
\left\|D f^{k}(x)(v)\right\| \geq c_{0} e^{k(\chi(\mu)-\varepsilon)}\|v\| \text { for every } v \in E_{x}^{u}, \\
\left\|D f^{-k}(x)(w)\right\| \leq c_{0} e^{k(-\chi(\mu)+\varepsilon)}\|w\| \text { for every } w \in E_{x}^{s} .
\end{gathered}
$$

This implies that

$$
\operatorname{Fix}\left(f^{k}\right) \cap K_{n} \subset \operatorname{SFix}\left(f^{k}, \alpha, c_{0}\right)
$$

for every $k \in \mathbb{N}$. It follows from (23) and the variational principle (14) that

$$
h_{\mu}(f)+\int_{\Lambda} \varphi d \mu-2 \varepsilon<h_{\mu_{n}}(f)+\int_{K_{n}} \varphi d \mu_{n} \leq P_{\text {top }}\left(f \mid K_{n}, \varphi\right) .
$$

Let $m, s \in \mathbb{N}$ such that $f^{m} \mid K_{n}$ is topologically conjugate to the full shift in $s$ symbols. Since $m P_{\text {top }}\left(f \mid K_{n}, \varphi\right)=P_{\text {top }}\left(f^{m} \mid K_{n}, S_{m} \varphi\right)$ (see [14, Theorem 9.8]), we can conclude that

$$
h_{\mu}(f)+\int_{\Lambda} \varphi d \mu-2 \varepsilon \leq \frac{1}{m} P_{\mathrm{top}}\left(f^{m} \mid K_{n}, S_{m} \varphi\right) .
$$


Recall that $S_{m} \varphi(x)=\sum_{i=0}^{m-1} \varphi\left(f^{i}(x)\right)$. It now follows from Proposition 1 and an elementary calculation that

$$
\begin{aligned}
h_{\mu}(f) & +\int_{\Lambda} \varphi d \mu-2 \varepsilon \\
& \leq \frac{1}{m} \lim _{k \rightarrow \infty} \frac{1}{k} \log \left(\sum_{x \in \operatorname{Fix}\left(f^{m k}\right) \cap K_{n}} \exp \left(\sum_{i=0}^{k-1} S_{m} \varphi\left(f^{i m}(x)\right)\right)\right) \\
& =\lim _{k \rightarrow \infty} \frac{1}{m k} \log \left(\sum_{x \in \operatorname{Fix}\left(f^{m k}\right) \cap K_{n}} \exp S_{m k} \varphi(x)\right) \\
& \leq \lim _{k \rightarrow \infty} \frac{1}{k} \log \left(\sum_{x \in \operatorname{Fix}\left(f^{k}\right) \cap K_{n}} \exp S_{k} \varphi(x)\right) .
\end{aligned}
$$

Combining (24) and (25) yields

$$
h_{\mu}(f)+\int_{\Lambda} \varphi d \mu-2 \varepsilon \leq \limsup _{k \rightarrow \infty} \frac{1}{k} \log \sum_{x \in \operatorname{SFix}\left(f^{k}, \alpha, c_{0}\right)} \exp S_{k} \varphi(x) .
$$

Recall that by $(10)$ the map $c \mapsto P_{\mathrm{SP}}(\varphi, \alpha, c)$ is non-decreasing as $c \rightarrow 0^{+}$. Since $\varepsilon>0$ was chosen arbitrarily the claimed statement follows.

Remarks. (i) We note that the hyperbolic horseshoes $K_{n}$ in the proof of Proposition 2 are in general not locally maximal $f$-invariant sets.

(ii) It follows from Ruelle's inequality that if $M$ is a surface, then $h_{\mu}(f)>0$ implies $\chi(\mu)>0$ and therefore, Proposition 2 holds for all measures with positive measure-theoretic entropy.

We now introduce a natural class of potentials. For $\varphi \in C(\Lambda, \mathbb{R})$ set

$$
\alpha(\varphi)=P_{\mathrm{top}}(\varphi)-\sup _{\nu \in \mathcal{M}} \int_{\Lambda} \varphi d \nu
$$

We say that a potential $\varphi$ belongs to $C^{f}(\Lambda, \mathbb{R})$ if

(a) $\alpha(\varphi)>0$;

(b) there exist $0<\delta(\varphi)<\alpha(\varphi)$ and a sequence $\left(\mu_{n}\right)_{n} \subset \mathcal{M}_{\mathrm{E}}$ of saddle type such that $\chi\left(\mu_{n}\right)>\delta(\varphi)$ for every $n \in \mathbb{N}$ and $h_{\mu_{n}}(f)+\int_{\Lambda} \varphi d \mu_{n} \rightarrow P_{\text {top }}(\varphi)$ as $n \rightarrow \infty$.

Remarks. (i) Note that $\alpha(\varphi) \geq 0$, and $\alpha(\varphi)>0$ if and only if $\varphi$ has no equilibrium state with zero entropy.

(ii) It follows from Ruelle's inequality and the variational principle that if $M$ is a surface, then property (b) follows from property (a).

(iii) We note that if $\varphi$ has a hyperbolic equilibrium measure $\mu_{\varphi}$, then we can simply choose the constant sequence $\mu_{n}=\mu_{\varphi}$ in (b).

(iv) It is easy to see that on higher dimensional manifolds the potential $\varphi=0$ does not always belong to $C^{f}(\Lambda, \mathbb{R})$. A simple counterexample is given below.

Example 1. Let $f=\left(f_{1}, f_{2}\right): S^{1} \times T^{2} \rightarrow S^{1} \times T^{2}$, where $f_{1}$ is a rotation on the circle and $f_{2}$ is an Anosov diffeomorphism on the 2-torus. Set $\Lambda=S^{1} \times T^{2}$ and $\varphi=0$. Then $\alpha(\varphi)=h_{\text {top }}(f)=h_{\text {top }}\left(f_{2}\right)>0$; nevertheless property (b) is not satisfied since $f$ does not have any hyperbolic measure. 
We now present the proof of our main result of Theorem 1 which was stated in the introduction.

Proof of Theorem 1. Let $0<\alpha<\delta(\varphi)$ and $0<\varepsilon<\delta(\varphi)-\alpha$. Let $\beta_{0}$ be defined as in (17). It follows from Theorem 2, (16) and (18) that

$$
P_{\mathrm{SP}}(\varphi, \alpha, c)=P_{\mathrm{SP}}\left(\varphi,\left[\alpha, \beta_{0}\right], c\right) \leq P_{\mathrm{top}}(\varphi)
$$

for all $0<c \leq 1$. It remains to prove that

$$
P_{\text {top }}(\varphi) \leq \lim _{c \rightarrow 0} P_{\mathrm{SP}}(\varphi, \alpha, c) .
$$

Since $\varphi \in C^{f}(\Lambda, \mathbb{R})$, there exist $n \in \mathbb{N}$ and $\mu_{n} \in \mathcal{M}_{\mathrm{E}}$ with $\chi\left(\mu_{n}\right)>\delta(\varphi)$ such that

$$
P_{\text {top }}(\varphi)-\varepsilon \leq h_{\mu_{n}}(f)+\int_{\Lambda} \varphi d \mu_{n}
$$

It follows from (26) that

$$
\alpha<P_{\text {top }}(\varphi)-\sup _{\nu \in \mathcal{M}} \int_{\Lambda} \varphi d \nu-\varepsilon \leq h_{\mu_{n}}(f)+\int_{\Lambda} \varphi d \mu_{n}-\sup _{\nu \in \mathcal{M}} \int_{\Lambda} \varphi d \nu \leq h_{\mu_{n}}(f) .
$$

Therefore, Proposition 2 implies

$$
h_{\mu_{n}}(f)+\int_{\Lambda} \varphi d \mu_{n} \leq \lim _{c \rightarrow 0} P_{\mathrm{SP}}(\varphi, \alpha, c) .
$$

Since $\varepsilon$ can be chosen arbitrary small, (27) and (28) imply (2). Finally, if $M$ is a surface, then by Ruelle's inequality property (b) in the definition of $C^{f}(\Lambda, \mathbb{R})$ holds for all $0<\delta(\varphi)<\alpha(\varphi)$. Thus, (2) holds for all $0<\alpha<\alpha(\varphi)$.

Remarks. (i) Note that Theorem 1 holds for every fixed $\alpha$ satisfying $0<\alpha<\delta(\varphi)$. Therefore, the topological pressure of $\varphi$ is entirely determined by the values of $\varphi$ on the saddle points of $f$.

(ii) As stated in the introduction Theorem 1 (and also Theorem 2) generalizes results of Chung and Hirayama [8]. They considered the entropy (i.e. $\varphi=0$ ) of surface diffeomorphisms, and our results allow more general potentials in $C^{f}(\Lambda, \mathbb{R})$ and also diffeomorphisms on higher dimensional manifolds. We note that we have used several ideas of [8] in our proofs. For related results in the case of $C^{1}$-maps on the interval or the circle we refer to [7].

The following example shows that the hypothesis $\alpha(\varphi)>0$ in Theorem 1 cannot be omitted.

Example 2. Let $f: \mathbb{R}^{2} \rightarrow \mathbb{R}^{2}$ be a $C^{2}$-diffeomorphism having a hyperbolic horseshoe $\widetilde{\Lambda}$ as well as an attracting fixed point $x$. Define $\Lambda=\widetilde{\Lambda} \cup\{x\}$. Moreover, let $\varphi \in C(\Lambda, \mathbb{R})$ with $\varphi \mid \widetilde{\Lambda}=0$ and $\varphi(x)>h_{\text {top }}(f \mid \Lambda)$. Then $\alpha(\varphi)=0$ and $P_{\text {top }}(\varphi)=\varphi(x)>\lim _{c \rightarrow 0} P_{\mathrm{SP}}(\varphi, \alpha, c)=h_{\text {top }}(f \mid \Lambda)$ for every $0<\alpha<h_{\text {top }}(f \mid \Lambda)$.

\section{Non-Continuous potentials - The volume PRessure}

It is well-known that if $\Lambda$ is a locally maximal hyperbolic set of a $C^{2}$ diffeomorphism $f: M \rightarrow M$, then the value of the topological pressure of the potential $\varphi^{u}=-\log |\operatorname{det} D f| E^{u} \mid$ has significant impact on the geometry of $\Lambda$ as well as on the dynamics of $f$ in a neighborhood of $\Lambda$. For example, the classical result of Bowen [5] states that $P_{\text {top }}\left(\varphi^{u}\right)=0$ if and only if $\Lambda$ is an attractor. In the case of more general systems, no such continuous $D f$-invariant splitting may exist, and 
therefore, one cannot even define the continuous potential $\varphi^{u}$ on $\Lambda$. To overcome this problem we introduce in this section a "potential" $\varphi^{u}$ defined only on a certain subset of $\Lambda$ and then consider the saddle point pressure of $\varphi^{u}$ rather than its topological pressure.

Let $M$ be a smooth Riemannian manifold and let $f: M \rightarrow M$ be a $C^{2}$ diffeomorphism. Suppose that $\Lambda \subset M$ is a compact locally maximal $f$-invariant set with $h_{\text {top }}(f \mid \Lambda)>0$. Define

$$
\chi(\Lambda) \stackrel{\text { def }}{=} \sup _{\mu} \chi(\mu)
$$

where the supremum is taken over all $\mu \in \mathcal{M}_{E}$ supported in $\Lambda$ which have at least one positive as well as one negative Lyapunov exponent. Since $h_{\text {top }}(f \mid \Lambda)>0$, the variational principle for the topological entropy and Ruelle's inequality imply that the supremum in the definition of $\chi(\Lambda)$ is not taken over the empty set. Note that $\chi(\Lambda)>0$ is equivalent to the existence of a measure $\mu \in \mathcal{M}_{E}$ of saddle type. We denote by $\mathcal{R}$ the set of Lyapunov regular points in $\Lambda$ (see Section 2.1 and [3] for details). Moreover, we define

$$
\mathcal{R}_{ \pm} \stackrel{\text { def }}{=}\left\{x \in \mathcal{R}: \lambda_{1}(x)<0<\lambda_{\operatorname{dim} M}(x)\right\}
$$

and

$$
\mathcal{R}_{\mathrm{H}} \stackrel{\text { def }}{=}\left\{x \in \mathcal{R}: \lambda_{l(x)}(x)<0<\lambda_{l(x)+1}(x) \text { for some } 1 \leq l(x)<\operatorname{dim} M\right\} .
$$

Clearly $\mathcal{R}_{\mathrm{H}} \subset \mathcal{R}_{ \pm}$. Let $\mu \in \mathcal{M}_{E}$ have at least one positive and one negative Lyapunov exponent. It follows from the multiplicative ergodic theorem that

$$
\lambda_{i}(x)=\lambda_{i}(\mu)
$$

for $\mu$-almost every $x \in \Lambda$. Denote by $\Lambda(\mu)$ the set of points in $\mathcal{R}_{ \pm}$satisfying (29). Hence $\mu(\Lambda(\mu))=1$ and, in particular, if $\mu$ is of saddle type, then $\Lambda(\mu) \subset \mathcal{R}_{\mathrm{H}}$. Given $x \in \mathcal{R}_{ \pm}$we define subspaces

$$
E_{x}^{s} \stackrel{\text { def }}{=} \bigoplus_{i: \lambda_{i}(x)<0} E_{x}^{i}, \quad E_{x}^{c} \stackrel{\text { def }}{=} \bigoplus_{i: \lambda_{i}(x)=0} E_{x}^{i} \quad \text { and } \quad E_{x}^{u} \stackrel{\text { def }}{=} \bigoplus_{i: \lambda_{i}(x)>0} E_{x}^{i} .
$$

Hence, $x \mapsto E_{x}^{u / s / c}$ are Borel measurable functions on $\Lambda(\mu)$ and form a $D f$-invariant splitting of the tangent bundle $T_{\Lambda(\mu)} M=E^{s} \oplus E^{c} \oplus E^{u}$. Moreover, if $\mu$ is of saddle type, then $E_{x}^{c}=\{0\}$ for $\mu$-almost every $x \in \Lambda$. We define

$$
\mathcal{L} \stackrel{\text { def }}{=} \bigcup_{\mu} \Lambda(\mu)
$$

where the union is taken over all measures $\mu \in \mathcal{M}_{E}$ having at least one positive and one negative Lyapunov exponent. Analogously, we define $\mathcal{L}_{H}=\bigcup_{\mu} \Lambda(\mu)$, where the union is taken over all measures $\mu \in \mathcal{M}_{E}$ of saddle type. We define $\varphi^{u}: \mathcal{L} \rightarrow \mathbb{R}$ by

$$
\varphi^{u}(x) \stackrel{\text { def }}{=}-\log |\operatorname{det} D f(x)| E_{x}^{u} \mid \text {. }
$$

We note that in general neither the splitting $T_{\mathcal{L}} M=E^{s} \oplus E^{c} \oplus E^{u}$, nor the splitting $T_{\mathcal{L}_{H}} M=E^{s} \oplus E^{u}$ can be continuously extended to $T_{\Lambda} M$, and therefore there is no continuous function $\varphi \in C(\Lambda, \mathbb{R})$ with $\varphi \mid \mathcal{L}=\varphi^{u}$ or $\varphi \mid \mathcal{L}_{H}=\varphi^{u}$. Clearly, $\operatorname{SPer}(f) \subset \mathcal{L}_{H}$, and thus, $\varphi^{u}(x)$ is well-defined for every $x \in \operatorname{SPer}(f)$. Let $0<\alpha$ and $0<c \leq 1$. Define

$$
Q_{\mathrm{SP}}\left(\varphi^{u}, \alpha, c, n\right) \stackrel{\text { def }}{=} \sum_{x \in \operatorname{SFix}\left(f^{n}, \alpha, c\right)} \exp S_{n} \varphi^{u}(x)
$$


if $\operatorname{SFix}\left(f^{n}, \alpha, c\right) \neq \emptyset$ and

$$
Q_{\mathrm{SP}}\left(\varphi^{u}, \alpha, c, n\right) \stackrel{\text { def }}{=} \exp \left(n \inf _{x \in \mathcal{L}} \varphi^{u}(x)\right)
$$

otherwise. We define

$$
P_{\mathrm{SP}}\left(\varphi^{u}, \alpha, c\right) \stackrel{\text { def }}{=} \limsup _{n \rightarrow \infty} \frac{1}{n} \log Q_{\mathrm{SP}}\left(\varphi^{u}, \alpha, c, n\right) .
$$

It follows that if $\operatorname{SFix}\left(f^{n}, \alpha, c\right) \neq \emptyset$ for some $n \in \mathbb{N}$, then $P_{\mathrm{SP}}\left(\varphi^{u}, \alpha, c\right)$ is entirely determined by the values of $\varphi^{u}$ on the saddle points of $f$. By $D f$-invariance of $E^{u}$ on $\mathcal{L}$, we conclude that

$$
\left.\left|\operatorname{det} D f^{n}(x)\right| E_{x}^{u}\right|^{-1}=\exp S_{n} \varphi^{u}(x)
$$

for all $x \in \mathcal{L}$. Moreover, if $x \in \mathcal{L}$, then

$$
\lim _{n \rightarrow \infty} \frac{1}{n} \log \left|\operatorname{det} D f^{n}(x)\right| E_{x}^{u} \mid=\sum_{i: \lambda_{i}(x)>0} \lambda_{i}(x) .
$$

In particular,

$$
\int_{\Lambda} \varphi^{u} d \mu=-\int_{\Lambda} \sum_{i: \lambda_{i}(x)>0} \lambda_{i}(x) d \mu(x)
$$

for every measure $\mu \in \mathcal{M}_{\mathrm{E}}$ of saddle type.

We now state a variational inequality for the above pressure.

Theorem 3. Let $f: M \rightarrow M$ be a $C^{2}$-diffeomorphism and let $\Lambda \subset M$ be a compact locally maximal $f$-invariant set. Let $0<\alpha$ such that $\operatorname{SFix}\left(f^{n}, \alpha, c_{0}\right) \neq \emptyset$ for some $n \in \mathbb{N}$ and some $0<c_{0} \leq 1$. Then

$$
\lim _{c \rightarrow 0} P_{\mathrm{SP}}\left(\varphi^{u}, \alpha, c\right) \leq \sup _{\nu}\left(h_{\nu}(f)+\int_{\Lambda} \varphi^{u} d \nu\right),
$$

where the supremum is taken over all measures $\nu \in \mathcal{M}_{\mathrm{E}}$ of saddle type with $\alpha \leq$ $\chi(\nu)$.

Proof. Since there is $n \in \mathbb{N}$ and $0<c_{0} \leq 1$ such that $\operatorname{SFix}\left(f^{n}, \alpha, c_{0}\right) \neq \emptyset$, it follows that $\chi(\Lambda)>0$; in particular, the supremum in (30) is not taken over the empty set. Let $\beta_{0}$ be defined as in (17) and let $0<c \leq c_{0}$. Analogously as in the proof of Theorem 2 we are able to construct a compact hyperbolic set $K=K_{\alpha, \beta_{0}, c} \subset \Lambda$ with

$$
\operatorname{Fix}\left(f^{n}\right) \cap K=\operatorname{SFix}\left(f^{n}, \alpha, c\right)
$$

for all $n \in \mathbb{N}$. Hence

$$
P_{\mathrm{SP}}\left(\varphi^{u}, \alpha, c\right)=\limsup _{n \rightarrow \infty} \frac{1}{n} \log \left(\sum_{x \in \operatorname{Fix}\left(f^{n}\right) \cap K} \exp S_{n} \varphi^{u}(x)\right) .
$$

Since $K$ is hyperbolic, the potential $\varphi_{K}^{u}=-\log |\operatorname{det} D f| E^{u} \mid: K \rightarrow \mathbb{R}$ is Hölder continuous. Therefore, Proposition 1 and (32) imply that

$$
P_{\mathrm{SP}}\left(\varphi^{u}, \alpha, c\right) \leq P_{\text {top }}\left(f \mid K, \varphi_{K}^{u}\right) .
$$

On the other hand, the variational principle gives

$$
P_{\text {top }}\left(f \mid K, \varphi_{K}^{u}\right)=\sup _{\nu}\left(h_{\nu}(f)+\int \varphi^{u} d \nu\right),
$$


where the supremum is taken over all $\nu \in \mathcal{M}_{\mathrm{E}}$ which are supported on $K$ (and which, in particular, are of saddle type). Moreover, by construction of $K$ we have that $\alpha \leq \chi(\nu)$ holds for these measures. We conclude that

$$
\lim _{c \rightarrow 0} P_{\mathrm{SP}}\left(\varphi^{u}, \alpha, c\right) \leq \sup _{\nu}\left(h_{\nu}(f)+\int \varphi^{u} d \nu\right),
$$

where the supremum is taken over all measures $\nu \in \mathcal{M}_{\mathrm{E}}$ of saddle type with $\alpha \leq$ $\chi(\nu)$.

Under the assumptions of Theorem 3 we call

$$
P_{\mathrm{SP}}\left(\varphi^{u}\right) \stackrel{\text { def }}{=} \lim _{\alpha \rightarrow 0} \lim _{c \rightarrow 0} P_{\mathrm{SP}}\left(\varphi^{u}, \alpha, c\right)
$$

the volume pressure of $f$.

Remark. We note that if $\Lambda$ is a locally maximal hyperbolic set such that $f \mid \Lambda$ is topologically mixing, then the volume pressure coincides with the topological pressure of the potential $\varphi_{\Lambda}^{u}=-\log |\operatorname{det} D f| E^{u} \mid: \Lambda \rightarrow \mathbb{R}$.

As an immediate consequence of the proof of Theorem 3 we obtain the following "inverse" variational inequality for the volume pressure.

Corollary 1. Let $f$ and $\Lambda$ be as in Theorem 3, and assume that $f$ has a saddle point in $\Lambda$. Then

$$
P_{\mathrm{SP}}\left(\varphi^{u}\right) \leq \sup _{K} P_{\text {top }}\left(f|K,-\log | \operatorname{det} D f\left|E^{u}\right|\right),
$$

where the supremum is taken over all compact hyperbolic sets $K \subset \Lambda$.

Corollary 1 immediately implies the following.

Corollary 2. Let $f$ and $\Lambda$ be as in Theorem 3, and assume that $f$ has a saddle point in $\Lambda$. Then

$$
P_{\mathrm{SP}}\left(\varphi^{u}\right) \leq \sup _{\nu}\left(h_{\nu}(f)+\int_{\Lambda} \varphi^{u} d \nu\right),
$$

where the supremum is taken over all measures $\nu \in \mathcal{M}_{\mathrm{E}}$ of saddle type.

\section{RATE OF ESCAPE FROM NEIGHBORHOODS AND DIMENSION OF INVARIANT SETS}

In this section we discuss relations between the attraction properties of the invariant set $\Lambda$ and the dimension of certain subsets of $\Lambda$.

Let $\Lambda \subset M$ be a compact locally maximal $f$-invariant set and let $U \subset M$ be an open neighborhood of $\Lambda$ such that $\Lambda=\bigcap_{n \in \mathbb{Z}} f^{n}(U)$. Given an open neighborhood $V \subset U$ of $\Lambda$ we define the upper (exponential) escape rate from $V$ by

$$
\bar{E}(V) \stackrel{\text { def }}{=} \limsup _{n \rightarrow \infty} \frac{1}{n} \log \left(\operatorname{vol} \bigcap_{k=0}^{n-1} f^{-k}(V)\right),
$$

where vol denotes the volume induced by the Riemannian metric on $M$. Analogously, we define the lower escape rate $\underline{E}(V)$ by replacing the limes superior in (33) with the limes inferior. By definition, we have that $\underline{E}(V) \leq \bar{E}(V) \leq 0$. Under the 
assumption that the upper escape rate is strictly negative we obtain a non-trivial upper bound for the upper box dimension of $\Lambda$ (see Theorem 4 below). Define

$$
s \stackrel{\text { def }}{=} \lim _{n \rightarrow \infty} \frac{1}{n} \log \left(\max _{x \in \Lambda}\left\|D f^{n}(x)\right\|\right) .
$$

Note that $s$ is well-defined. This follows from the sub-additivity of the sequence $\left(\varphi_{n}\right)_{n}$ given by $\varphi_{n}=\log \left(\max _{x \in \Lambda}\left\|D f^{n}(x)\right\|\right)$ (see e.g. [14]).

Theorem 4. Let $f: M \rightarrow M$ be a $C^{2}$-diffeomorphism, and let $\Lambda \subset M$ be a compact locally maximal $f$-invariant set containing a point with a positive Lyapunov exponent. Then

$$
\overline{\operatorname{dim}}_{\mathrm{B}} \Lambda \leq \operatorname{dim} M+\frac{\bar{E}(V)}{s}
$$

In particular, if $\bar{E}(V)<0$, then $\overline{\operatorname{dim}}_{\mathrm{B}} \Lambda<\operatorname{dim} M$.

Proof. First, we note that since $\Lambda$ contains a point with a positive Lyapunov exponent, it follows that $s>0$. Let $\delta>0$. By a simple continuity argument there exist $\varepsilon>0$ and $n(\delta) \in \mathbb{N}$ such that for all $y \in B(\Lambda, \varepsilon) \stackrel{\text { def }}{=} \bigcup_{x \in \Lambda} B(x, \varepsilon)$ we have

$$
\left\|D f^{n(\delta)}(y)\right\|<\exp (n(\delta)(s+\delta)) .
$$

From now on we consider the map $g=f^{n(\delta)}$. Note that $\Lambda$ is also a compact invariant set of $g$. It follows from (33) and (34) that $\bar{E}_{g}(V) \leq n(\delta) \bar{E}_{f}(V)$ and $s_{g}=n(\delta) s_{f}$. Therefore, it suffices to prove (35) for $g$. We continue to use the notation $s$ and $\bar{E}(V)$ for $g$ instead of $f$.

Set $V=B(\Lambda, \varepsilon)$. By making $\varepsilon$ smaller if necessary we can assure that $V \subset U$. It follows from the definition of $\bar{E}(V)$ that if $n$ is sufficiently large, then

$$
\operatorname{vol}\left(\bigcap_{k=0}^{n-1} f^{-k}(V)\right)<\exp (n(\bar{E}(V)+\delta)) \text {. }
$$

For $n \in \mathbb{N}$ we define real numbers

$$
r_{n}=\frac{\varepsilon}{\exp (n(s+\delta))}
$$

and neighborhoods $B_{n}=B\left(\Lambda, r_{n}\right)$ of $\Lambda$. Let $y \in B_{n}$. Then there exists $x \in \Lambda$ with $d(x, y)<r_{n}$. An elementary induction argument in combination with the mean-value theorem implies $d\left(g^{i}(x), g^{i}(y)\right)<\varepsilon$ for all $i \in\{0, \ldots, n-1\}$. Hence $B_{n} \subset \bigcap_{k=0}^{n-1} g^{-k}(V)$, and (36) implies that

$$
\operatorname{vol}\left(B_{n}\right)<\exp (n(\bar{E}(V)+\delta))
$$

for sufficiently large $n$. Let us recall that for $t \in[0, \operatorname{dim} M]$ the $t$-dimensional upper Minkowski content of a relatively compact set $A \subset M$ is defined by

$$
M^{* t}(A)=\limsup _{\rho \rightarrow 0} \frac{\operatorname{vol}\left(A_{\rho}\right)}{(2 \rho)^{\operatorname{dim} M-t}},
$$


where $A_{\rho}=\{y \in M: \exists x \in A: d(x, y) \leq \rho\}$. Let $t \in[0, \operatorname{dim} M]$ and $\rho_{n}=\frac{r_{n}}{2}$ for all $n \in \mathbb{N}$. Then we have

$$
\begin{aligned}
& M^{* t}(\Lambda)=\limsup _{\rho \rightarrow 0} \frac{\operatorname{vol}\left(\Lambda_{\rho}\right)}{(2 \rho)^{\operatorname{dim} M-t}} \\
& \leq \limsup _{n \rightarrow \infty} \frac{\operatorname{vol}\left(\Lambda_{\rho_{n}}\right)}{\left(2 \rho_{n+1}\right)^{\operatorname{dim} M-t}} \leq \limsup _{n \rightarrow \infty} \frac{\operatorname{vol}\left(B_{n}\right)}{\left(r_{n+1}\right)^{\operatorname{dim} M-t}} \\
& \leq \frac{\exp ((\operatorname{dim} M-t)(s+\delta))}{\varepsilon^{\operatorname{dim} M-t}} \lim _{n \rightarrow \infty}(\exp ((\operatorname{dim} M-t)(s+\delta)+\bar{E}(V)+\delta))^{n} .
\end{aligned}
$$

Assume $t>\operatorname{dim} M+(\bar{E}(V)+\delta) /(s+\delta)$. Then

$$
\exp ((\operatorname{dim} M-t)(s+\delta)+\bar{E}(V)+\delta)<1,
$$

which implies $M^{* t}(\Lambda)=0$. Hence $t \geq \overline{\operatorname{dim}}_{\mathrm{B}} \Lambda$ (see [11]). Finally, the fact that $\delta$ was arbitrary completes the proof.

Remarks. (i) In the proof of Theorem 4 we have used similar ideas as in [13] in the context of hyperbolic sets.

(ii) It is easy to see that the analog of Theorem 4 holds for the lower box dimension with $\bar{E}(V)$ replaced by $\underline{E}(V)$ in $(35)$.

(iii) The dimension estimate in Theorem 4 remains true when $f$ is locally a $C^{1}$-diffeomorphism.

It is a result of Young $[15$, Theorem $4(1)]$ that

$$
\sup _{\nu \in \mathcal{M}_{\mathrm{E}}}\left(h_{\nu}(f)+\int_{\Lambda} \varphi^{u} d \nu\right) \leq \underline{E}(V) \leq \bar{E}(V) \leq 0 .
$$

Therefore, Theorem 3 implies that if $P_{\mathrm{SP}}\left(\varphi^{u}\right)=0$, then $\underline{E}(V)=\bar{E}(V)=0$ and thus the volume of $\bigcap_{k=0}^{n-1} f^{-k}(V)$ is shrinking at a sub-exponential rate. It is a classical result of Bowen [5] that in the case when $\Lambda$ is a hyperbolic set of $f$ then

$$
\sup _{\nu \in \mathcal{M}_{\mathrm{E}}}\left(h_{\nu}(f)+\int_{\Lambda} \varphi^{u} d \nu\right)=\underline{E}(V)=\bar{E}(V)
$$

and thus the volume of $\bigcap_{k=0}^{n-1} f^{-k}(V)$ shrinks at an exponential rate (in which case $\Lambda$ is an attractor) if and only if $P_{\mathrm{SP}}\left(\varphi^{u}\right)=0$.

Baladi et al. [1] give an example of a compact locally maximal $f$-invariant set $\Lambda$ of a $C^{\infty}$-surface diffeomorphism and an invariant measure $\mu$, supported on $\Lambda$, which attains the supremum in (38) with the property that $h_{\mu}(f)+\int_{\Lambda} \varphi^{u} d \mu<\bar{E}(V)=0$ for an arbitrarily small neighborhood $V$ of $\Lambda$ (another example is given by the "figure-8 attractor"; see [9, p. 140]). Obviously, in such a situation Theorem 4 does not provide a non-trivial upper bound for the box dimension of $\Lambda$. The following example shows that in general such a non-trivial upper bound does not exist.

Example 3. Let $M$ be a smooth compact surface and let $f: M \rightarrow M$ be a $C^{2}$ diffeomorphism whose non-wandering set is a horseshoe $K$. Define $\Lambda=M$. Then $\overline{\operatorname{dim}}_{\mathrm{B}} \Lambda=\operatorname{dim} M=2$ and $\bar{E}(V)=0$, however $P_{\mathrm{SP}}\left(\varphi^{u}\right)=P_{\text {top }}\left(f \mid K, \varphi^{u}\right)<0$.

We end the paper with the following

Open problem. Does $P_{\mathrm{SP}}\left(\varphi^{u}\right)<0$ imply $\overline{\operatorname{dim}}_{\mathrm{B}} \overline{\operatorname{SPer}(f)}<\operatorname{dim} M$ ?

\section{ACKNOWLEDGMENT}

We would like to thank the referee for several comments and improvements. 


\section{REFERENCES}

[1] V. Baladi, Ch. Bonatti, and B. Schmitt, Abnormal escape rates from nonuniformly hyperbolic sets, Ergodic Theory Dynam. Systems 19 (1999), 1111-1125. MR1721612 (2000i:37035)

[2] L. Barreira, A non-additive thermodynamic formalism and applications to dimension theory of hyperbolic dynamical systems, Ergodic Theory Dynam. Systems 16 (1996), 871-927. MR1417767 (98a:58124)

[3] L. Barreira and Y. Pesin, Smooth ergodic theory and nonuniformly hyperbolic dynamics, in Handbook of Dynamical Systems 1B, B. Hasselblatt and A. Katok eds., Elsevier, 2006. MR2186242 (2007c:37023)

[4] C. Bonatti, L. Díaz, and E. Pujals, A $C^{1}$-generic dichotomy for diffeomorphisms: weak forms of hyperbolicity or infinitely many sinks of sources, Ann. Math. 158 (2003), 355-418. MR2018925

[5] R. Bowen, Equilibrium states and the ergodic theory of Anosov diffeomorphisms, Lecture Notes in Mathematics 470, Springer, 1975. MR0442989 (56:1364)

[6] R. Bowen and D. Ruelle, The ergodic theory of Axiom A flows, Invent. Math. 29 (1975), 181-202. MR0380889 (52:1786)

[7] Y. M. Chung, Expanding periodic orbits with small exponents, J. Difference Equ. Appl. 9 (2003), 337-341. MR1990340 (2004f:37026)

[8] Y. M. Chung and M. Hirayama, Topological entropy and periodic orbits of saddle type for surface diffeomorphisms, Hiroshima Math. J. 33 (2003), 189-195. MR1997693 (2004g:37055)

[9] A. Katok, Lyapunov exponents, entropy and periodic orbits for diffeomorphisms, Publ. Math., Inst. Hautes Étud. Sci. 51 (1980), 137-173. MR573822 (81i:28022)

[10] A. Katok and B. Hasselblatt, Introduction to the Modern Theory of Dynamical Systems, Encyclopedia of Mathematics and Its Applications 54, Cambridge University Press, 1995. MR1326374 (96c:58055)

[11] P. Mattila, Geometry of sets and measures in Euclidean spaces. Fractals and rectifiability, Cambridge University Press, 1995. MR1333890 (96h:28006)

[12] Y. Pesin, Dimension theory in dynamical systems: Contemporary Views and applications, Lectures in Mathematics, Chicago University Press, 1997. MR1489237 (99b:58003)

[13] R. Shafikov and Ch. Wolf, Stable sets, hyperbolicity and dimension, Discrete Contin. Dynam. Systems 12 (2005), 403-412. MR2119247 (2006j:37035)

[14] P. Walters, An introduction to ergodic theory, Graduate Textsin Mathematics 79, Springer, 1981. MR648108 (84e:28017)

[15] L.-S. Young, Large deviations in dynamical systems, Trans. Amer. Math. Soc. 318 (1990), 525-543. MR975689 (90g:58069)

Max-Planck-Institut für Physik komplexer Systeme, Nöthnitzer Str. 38, D-01187 Dresden, Germany - And - Institut für Physik, TU Chemnitz, D-09107 Chemnitz, GerMANY

E-mail address: gelfert@pks.mpg.de

$U R L:$ http://www.pks.mpg.de/ gelfert/

Department of Mathematics, Wichita State University, Wichita, Kansas 67260

E-mail address: cwolf@math.wichita.edu

URL: http://www.math.wichita.edu/ cwolf/ 